\title{
I calciomimetici nell'iperparatiroidismo primitivo e secondario
}

\author{
F. Locatelli, G. Pontoriero, M. Limardo \\ Dipartimento di Nefrologia, Dialisi e Trapianto, Ospedale A. Manzoni, Lecco
}

Iperparatiroidismo primitivo (IPTP) e secondario (IPTS) sono patologie delle paratiroidi caratterizzate da elevata sintesi e rilascio in circolo di paratormone (PTH) con conseguente alterazione del metabolismo calcio-fosforo.

In particolare nell'IPTP, come descritto estesamente nel capitolo dedicato alla fisiopatologia di questo disordine endocrino, l'iperattività ghiandolare è dovuta nella gran parte dei casi ad adenoma; meno frequente, è invece l'evenienza di iperplasia o carcinoma delle paratiroidi. Il quadro d'esordio della malattia, tradizionalmente caratterizzato da ipercalcemia, ipercalciuria, nefrolitiasi, e manifestazioni ossee, gastro- intestinali e neuromuscolari, è sostanzialmente cambiato negli ultimi anni. È sempre più frequente, infatti, l'identificazione di un quadro d'iperparatiroidismo primitivo a partire da un riscontro occasionale agli esami ematochimici di un modesto incremento dei livelli di calcemia in un paziente peraltro asintomatico.

L'IPTS è un disordine molto comune nei pazienti affetti da malattia renale cronica (CKD) che si sviluppa come meccanismo adattativo per controbilanciare le alterazioni dell'omeostasi calcio-fosforica indotte dalla progressiva perdita della funzione renale ma che alla lunga provoca severe conseguenze a livello sistemico. Come ben illustrato nel capitolo dedicato alla fisiopatologia dell'IPTS, considerevoli progressi nella conoscenza dei meccanismi fisiopatologici dell'IPTS sono stati fatti in anni recenti grazie alla scoperta dei recettori della vitamina D (VDR), del calcio (CaSR) e dell'ormone FGF23 (Fibroblastic Growth Factor 23) e allo studio dei meccanismi di regolazione della sintesi del calcitriolo. Nonostante ciò, è ancora controverso il fattore scatenante lo sviluppo dell'IPTS. Durante la naturale evoluzione della $\mathrm{CKD}$, il progressivo declino della funzione renale determina una sostanziale riduzione della produzione di calcitriolo e dell'escrezione renale di fosfati, con conseguente aumento dei livelli plasmatici di fosforo (P). Alcuni Autori ritengono che il fattore scatenante l'IPTS sia il deficit di calcitriolo, altri, invece, attribuiscono un ruolo determinante alla ritenzione di fosforo. Il ruolo svolto da ciascuno di questi fattori e le loro complesse interazioni sono illustrati nel capitolo dedicato alla fisiopatologia dell'IPTS. In particolare, un ruolo centrale nella genesi dell'IPTS è svolto dai livelli plasmatici di calcio $(\mathrm{Ca})$, che agiscono sulla secrezione del PTH attraverso il CaSR: elevati livelli di Ca inibiscono la sintesi e la secrezione di PTH; al contrario, bassi livelli di $\mathrm{Ca}$ inducono la produzione ed il rilascio in circolo di PTH. Mutazioni del gene del CaSR, a loro volta, possono essere responsabili di IPTP familiare, come illustrato nel capitolo dedicato alla fisiopatologia e trattamento delle forme familiari dell'IPTP. Sebbene iperparatiroidismo primitivo e secondario abbiano una diversa eziopatogenesi e manifestazioni tipiche (ossee e renali nel caso dell'IPTP, osteodistrofia renale nel caso dell'IPTS), entrambe espongono i pazienti ad un aumentato rischio di morbidità e mortalità cardiovascolare. I pazienti affetti da insufficienza renale cronica in trattamento dialitico hanno una aspettativa di vita ridotta da un-terzo a un-sesto rispetto alla popolazione generale, e le patologie cardiache rappresentano circa la metà delle cause di morte in questi pazienti $(1,2)$.

Le alterazioni del metabolismo Ca-P correlate all'IPTS possono giocare un ruolo determinante nella patologia cardiovascolare, favorendo lo sviluppo di calcificazioni vascolari e cardiache $(3,4)$ che possono essere aggravate anche da una non attenta terapia con vitamina $D$ e sali di calcio utilizzate proprio per contrastare le altera- 
zioni biochimiche legate all'IPTS.

Ampi studi osservazionali hanno evidenziato che nei pazienti in trattamento dialitico elevati livelli plasmatici di $\mathrm{PTH}, \mathrm{Ca}, \mathrm{P}$ e di prodotto calcio-fosforo (Ca-P) sono associati ad un aumentato rischio di morte (5), ma anche nei pazienti con insufficienza renale in trattamento conservativo bastano modesti incrementi dei livelli di fosforemia per determinare un significativo incremento del rischio di morte (6). In particolare, Block ha riportato che il rischio relativo di morte associato alle alterazioni del metabolismo minerale è nell'insieme pari al $17.5 \%$ e il maggiore determinante è l'iperfosforemia. Il ruolo preponderante del fosforo come fattore associato alla mortalità nei pazienti in emodialisi è stato confermato anche da uno studio prospettico condotto su 385 pazienti che hanno iniziato la dialisi tra il 1990 e il 2001: anche livelli di fosforemia modestamente aumentati (da 5.0 a $6.5 \mathrm{mg} / \mathrm{dL}$ [1.6-2.1 $\mathrm{mmol} / \mathrm{L}]$ ) sono risultati associati ad un rischio relativo di morte quasi doppio rispetto al gruppo di riferimento con fosforemia nella norma (7). In uno studio di coorte della durata di due anni condotto su un'ampia casistica (58.058 pazienti in emodialisi) è stato confermato il ruolo dei livelli plasmatici di Ca, P e PTH quali fattori predittivi di morte, tuttavia, quando nel modello statistico venivano considerati indicatori dello stato nutrizionale e infiammatorio, l'associazione tra mortalità ed alterazioni del metabolismo minerale si attenuava notevolmente (8) (ciò conferma come i dati di associazione possano essere fallaci perché, per quanto frutto di analisi ben strutturate, non possono tenere conto di tutti i possibili fattori confondenti).

Rilevante è anche l'interesse sulla relazione tra vitamina $\mathrm{D}$ e mortalità: la mortalità a 90 giorni dall'inizio della dialisi in 984 pazienti è risultata più elevata nei pazienti che prima della dialisi presentavano bassi livelli sierici di 25-idrossivitamina D (9). L'aumentato rischio di mortalità per ridotti livelli sierici di 25-idrossivitamina $\mathrm{D}$ è stato confermato anche per pazienti con un grado più modesto di danno renale (CKD stadio 25) (10).

L'interesse alla relazione tra morbilità-mortalità ed alterazione del metabolismo minerale è dettato dalla documentata possibilità di correggere queste alterazioni almeno in parte, con la terapia medica o chirurgica. Su questa base, nel 2003 le linee guida per il trattamento dell'IPTS della National Kidney Foundation-Kidney Disease Outcomes Quality Initiative (NKF-K/DOQI) hanno definito quali dovessero essere i livelli di PTH, $\mathrm{Ca}$ e $\mathrm{P}$ a seconda del grado di CKD (11).

Una recente analisi dello studio DOPPS (Dialysis Outcomes and Practice Patterns Study) I, II e III (12), ha considerato i dati raccolti dal 1996 al 2007, in un'ampia casistica internazionale di 25.588 pazienti in trattamento emodialitico. D'interesse in quest'analisi è la documentazione di un progressivo miglioramento nel tempo del controllo delle alterazioni del metabolismo minerale, in particolare per quanto riguarda i livelli di fosforemia. Questo risultato positivo può essere attribuito sia alla disponibilità di nuovi farmaci e nuove strategie terapeutiche sia all'azione proattiva ed educativa dell'ampia diffusione di linee guida quali le K/DOQI stesse.

Le prime linee guida internazionali per la terapia della malattia renale cronica e dei disordini del metabolismo minerale e dell'osso (CKD-Mineral and Bone Disorder, CKD-MBD) sono attese a breve dal Kidney Disease - Improving Global Outcomes (KDIGO). L'adozione di queste raccomandazioni ha l'obiettivo di migliorare la comunicazione, facilitare il clinico nelle sue scelte e promuovere l'implementazione delle linee guida o suggerimenti per la pratica clinica a livello locale.

La terapia tradizionale dell'IPTS comprende la modulazione del bilancio di Ca e P mediante la dieta, l'integrazione orale di supplementi di calcio ai pasti per sfruttarne anche l'azione chelante del fosforo, la modulazione della concentrazione di calcio nel bagno di dialisi, l'uso di chelanti del fosforo (a base di calcio e non), della vitamina $\mathrm{D}$ e dei suoi analoghi. La terapia con metaboliti attivi della vitamina $\mathrm{D}$ ha rappresentato in passato il solo mezzo, oltre all'intervento di paratiroidectomia, per ridurre i livelli di PTH nei pazienti affetti da IPTS. Tuttavia l'aumento dei livelli plasmatici di Ca e P dopo il trattamento con vitamina D ne limitano spesso l'impiego.

Alle terapie tradizionali, negli ultimi anni, si è aggiunta la disponibilità di nuovi chelanti del $\mathrm{P}$ non a base di calcio, come sevelamer $\mathrm{HCl}$ e carbonato di lantanio; mentre altri come MCI-196, sevelamer carbonato, e nuovi chelanti a base di ferro (come SBR759) sono attualmente in fase di registrazione o in studio. Vi è poi una nuova classe di farmaci, i calciomimetici, che ha profondamente modificato l'approccio al trattamento dell'IPTS.

Come descritto nel capitolo dedicato all'uso dei calciomimetici nell'IPTS, questa nuova classe di farmaci è in grado di modulare allostericamente l'attività dei recettori del calcio, i principali regolatori della secrezione del PTH. In presenza dei calciomimetici, il fisiologico effetto del calcio endogeno è potenziato e la secrezione di PTH inibita. Il Cinacalcet è il primo ed unico calciomimetico attualmente in uso per il trattamento clinico dell'iperparatiroidismo primitivo e secondario. L'uso del calciomimetico, per quanto riguarda l'IPTP, è 
limitato a quei pazienti in cui la terapia chirurgica non sia possibile o conveniente o nei quali la paratiroidectomia abbia fallito il suo scopo. Inoltre, come illustrato nel capitolo dedicato alle forme familiari di IPTP, il Cinacalcet può essere considerato tra $\mathrm{i}$ possibili trattamenti alternativi per i pazienti affetti da IPTP familiare che non rispondono ai criteri per la paratiroidectomia e/o con IPTP persistente o ricorrente, sebbene i dati sull'impiego del Cinacalcet in queste forme siano estremamente limitati, data la loro rarità.

L'efficacia di Cinacalcet nel trattamento dell'IPTS è stata dimostrata in studi clinici randomizzati, controllati in doppio cieco di fase III che hanno incluso un ampio numero di pazienti negli Stati Uniti, Europa, Canada e Australia $(13,14)$. Questi studi sono presentati in dettaglio nel capitolo Uso dei calciomimetici nell'iperparatiroidismo secondario. Nel loro insieme, questi studi hanno dimostrato che Cinacalcet, in associazione alla terapia tradizionale, è efficace nel ridurre i livelli plasmatici di PTH nei pazienti con CKD 5 in trattamento dialitico ed IPTS non controllato (PTH $>300 \mathrm{pg} / \mathrm{mL}[31.8 \mathrm{pmol} / \mathrm{L}])$. Inoltre, rispetto alla terapia tradizionale, il Cinacalcet ha ridotto significativamente e simultaneamente le concentrazioni plasmatiche di PTH, Ca, P e il prodotto Ca-P. Pertanto i calciomimetici migliorano il controllo dell'IPTS e consentono di conseguire con più agio ed in un maggior numero di pazienti i target delle linee guida per PTH, $\mathrm{Ca}, \mathrm{P}$ e prodotto Ca-P (15).

È di rilievo il fatto che la riduzione dei livelli plasmatici di PTH è indipendente dalla severità della malattia: il PTH si riduce di circa il 50\% indipendentemente dai suoi livelli iniziali. Inoltre, rispetto al gruppo di controllo, la frequenza di soggetti che raggiungeva il target di PTH fissato dallo studio era maggiore nei pazienti trattati con Cinacalcet, indipendentemente dall'uso concomitante di vitamina $\mathrm{D}$ e/o chelanti del fosforo a base di calcio (16). Questo risultato conferma che Cinacalcet può essere utilizzato sia da solo che in associazione con la terapia tradizionale.

Alcuni studi hanno valutato l'associazione di Cinacalcet e basse dosi di vitamina $\mathrm{D}$, dimostrando che l'impiego di Cinacalcet permette di ridurre il rischio di ipercalcemia ed iperfosforemia associati all'uso della vitamina $\mathrm{D}$ e nel contempo migliora il controllo dei livelli plasmatici di PTH, Ca e P $(17,18)$.

L'efficacia di Cinacalcet non sembra diminuire con la durata del trattamento; gli effetti della terapia con Cinacalcet si sono infatti mantenuti invariati in pazienti trattati per 3-4 anni $(19,20)$ e non vi è stata comparsa di tolleranza o resistenza al farmaco. Questi requisiti sono fondamentali considerando che la terapia dell'IPT è una terapia di lunga durata in pazienti affetti da una patologia cronica.

Alcuni hanno paragonato la terapia con Cinacalcet per la sua efficacia ad una specie di "paratiroidectomia farmacologica”. Ciò che ne può limitare l'uso sono i possibili effetti collaterali. Circa il 30\% dei pazienti può manifestare nausea e vomito e questo può ridurre la compliance e in alcuni casi portare alla sospensione del farmaco. Il meccanismo fisiopatologico alla base di questi effetti collaterali non è noto, tuttavia si può ipotizzare che esso sia mediato dall'azione dei calciomimetici sui CaSR presenti nelle cellule dell'apparato gastroenterico. Dato che i CaSR sono presenti sulle cellule di vari apparati dell'organismo va tenuta presente l'eventualità che i calciomimetici possano determinare, sul lungo periodo, effetti indesiderati non ancora noti e ciò suggerisce la necessità di una attenta farmaco-vigilanza, come peraltro per tutti i farmaci di recente acquisizione nella pratica clinica.

Come descritto nel capitolo dedicato alla gestione dell'IPTS nei pazienti affetti da malattia renale cronica in terapia conservativa (CKD stadio 3 e 4), l'IPTS è una delle complicanze più frequenti in questi pazienti. Tuttavia, spesso non è adeguatamente riconosciuto e trattato. È ipotizzabile che un significativo miglioramento dell'IPTS sia possibile se efficaci interventi farmacologici fossero messi in atto più precocemente durante lo sviluppo della malattia renale. In questo ambito, le caratteristiche farmacologiche del Cinacalcet sono di interesse, data la sua documentata capacità di prevenire e ridurre, nel modello animale, l'iperplasia delle ghiandole paratiroidi e gli effetti negativi dell'IPTS sull'osso e le calcificazioni vascolari.

Uno studio di recente pubblicazione (21) ha mostrato che Cinacalcet determina nei pazienti con CKD stadio 3 - 4 una riduzione dei livelli di PTH, paragonabile a quella che si osserva nei pazienti in dialisi. Tuttavia, diversamente dai pazienti in dialisi, la concentrazione plasmatica di fosforo tende ad aumentare e la riduzione dei livelli di calcemia è più marcata e frequente. Questi fenomeni sono da attribuire all'inibizione dell'attività paratiroidea che in fase conservativa, in cui esiste una funzione renale sia pure ridotta, ha come conseguenza un aumento del riassorbimento tubulare del fosforo ed una riduzione del riassorbimento renale di calcio. Questi risultati hanno affievolito l'entusiasmo per l'uso dei calciomimetici in fase conservativa e posto la necessità di pensare, per questa fase, un diverso paradigma terapeutico (bassi dosaggi di Cinacalcet a rinforzo di una terapia già instaurata con metaboliti attivi della vitamina $\mathrm{D}$ e/o sali di calcio) rispetto a quello usato in dialisi. 
La persistenza di IPTS (definito come livelli plasmatici di PTH $>2.5$ volte i limiti superiori della norma o la necessità di paratiroidectomia) ha una prevalenza di circa il $17 \%$ nei 4 anni dopo il trapianto di rene (22) con una frequenza di paratiroidectomia pari a 8.9 per 1000 anni-paziente a rischio (23). Come discusso nel capitolo Iperparatiroidismo, Calciomimetici e trapianto rena$l e$, i calciomimetici potrebbero diventare una terapia alternativa nei pazienti portatori di trapianto renale candidati alla paratiroidectomia, anche se non c'è l'esperienza per valutare i risultati di questa terapia nel lungo termine. I dati disponibili provengono da studi condotti su un ridotto numero di pazienti e con periodi di osservazione brevi (24-26). In questi studi Cinacalcet ha ridotto le concentrazioni di calcio e PTH, mentre le concentrazioni medie di fosforo sierico sono aumentate o rimaste invariate. Ulteriori studi sono però necessari per valutare eventuali effetti a lungo termine in questi pazienti, soprattutto dal punto di vista della sicurezza.

Alcune domande rimangono aperte per quanto riguarda la terapia con calciomimetici, in particolare per quanto riguarda l'impatto sull'osso e sulle calcificazioni vascolari. È possibile infatti che un migliore controllo dei parametri biochimici del metabolismo minerale si traduca in un miglioramento dei risultati clinici, ma questi aspetti devono ancora essere ulteriormente studiati.

È importante considerare che spesso è necessario combinare l'uso di calciomimetico e vitamina D e/o dei chelanti a base di calcio per compensare una eccessiva riduzione dei livelli plasmatici di calcio o per ridurre maggiormente i livelli di PTH. In questi casi, un possibile bilancio positivo del calcio, in particolare se associato ad una eccessiva inibizione del turn-over osseo (malattia adinamica dell'osso), può aumentare il rischio di calcificazioni cardiovascolari.
Studi clinici in corso (come ADVANCE ed EVOLVE) hanno l'obiettivo di valutare risultati clinici nel lungo termine e determinare se i calciomimetici potranno in futuro modificare la morbidità e la mortalità legata all'IPTS.

Nel 2008 Cinacalcet è stato approvato in Italia anche per il trattamento dei pazienti con IPTP nei quali la paratiroidectomia, pur essendo indicata sulla base dei valori sierici di calcio e PTH, non è possibile per controindicazione alla chirurgia o rifiuto da parte del paziente, in particolare nelle forme asintomatiche con calcemia borderline ma nelle quali nel lungo termine potrebbero esservi complicanze renali, ossee e cardiovascolari. In questo contesto, la disponibilità di un farmaco in grado di controllare i livelli di PTH e calcio può essere un'importante opzione terapeutica, come evidenziato dagli studi condotti su un pur limitato numero di soggetti $(27,28)$ che vengono discussi nel capitolo dedicato alla terapia dell'IPTP.

Cinacalcet è risultato infine efficace nel ridurre la calcemia in pazienti con carcinoma paratiroideo inoperabile (29).

Concludendo, i calciomimetici rappresentano un nuovo valido strumento nell'armamentario medico per il trattamento dell'iperparatiroidismo secondario e primitivo la cui gestione è spesso complessa.

Indirizzo degli Autori:

Prof. Francesco Locatelli

Dipartimento di Nefrologia, Dialisi e Trapianto

Ospedale A. Manzoni

Via dell'Eremo, 9/11

23900 Lecco

f.locatelli@ospedale.lecco.it

\section{Bibliografia}

1. Excerpts from the United States Renal Data System 2004 Annual Data Report: Atlas of End-Stage Renal Disease in the United States. Am J Kidney Dis 2005; 45 (Suppl.1): A 5-7, S1-280.

2. Locatelli F, Bommer J, London GM, et al. Cardiovascular disease determinants in chronic renal failure: clinical approach and treatment. Nephrol Dial
Transplant 2001; 16: 459-68.

3. Goodman WG, Goldin J, Kuizon BD, et al. Coronaryartery calcification in young adults with end-stage renal disease who are undergoing dialysis. N Engl J Med 2000; 342: 1478-83.

4. Ganesh SK, Stack AG, Lewin NW, et al. Association of elevated serum $\mathrm{PO}(4), \mathrm{CaPO}$ (4) product, and parathyroid hormone with cardiac mortality risk in chronic hemodialysis patients. J Am Soc Nephrol 2001; 12: 2131-8. 
5. Block GA, Klassen P, Lazarus JM, et al. Mineral metabolism, mortality, and morbidity in maintenance hemodialysis. J Am Soc Nephrol 2004; 15: 2208-18.

6. Kestenbaum B, Sampson JN, Rudser KD, et al. Serum phosphate levels and mortality risk among people with chronic kidney disease. J Am Soc Nephrol 2005; 16: 5208.

7. Rodriguez Benot A, Martin Malo A, Alvarez Lara MA, Rodriguez M, Ajama P. Mild hyperphosphatemia and mortality in hemodialysis patients. Am J Kidney Dis 2005; 46(1): 68-77.

8. Kalantar Zadeh K, Kuwae N, Regidor DL, et al. Survival predictability of time-varying indicators of bone disease in maintenance hemodialysis patients. Kidney Int 2006; 70(4): 771-80.

9. Wolf M, Shah A, Gutierrez O, et al. Vitamin D levels and early mortality among incident hemodialysis patients. Kidney Int 2007; 72(8): 1004-13.

10. Ravani P, Malberti F, Tripepi G, et al. Vitamin D levels and patients outcome in chronic kidney disease. Kidney Int 2009; 75: 88-95.

11. National Kidney Foundation: Bone metabolism and disease in chronic kidney disease. Am J Kidney Dis 2003; 42 (Suppl. 3): S1-202.

12. Tentori F, Blayney MJ, Abert JM, et al. Mortality risk for dialysis patients with different levels of serum calcium, phosphorus and PTH: the Dialysis and Practice Pattern Study (DOPPS). Am J Kidney Dis 2008; 52: 519-30.

13. Block GA, Martin KJ, De Francisco AL, et al. Cinacalcet for secondary hyperparathyroidism in patients receiving hemodialysis. N Engl J Med 2004; 8; 350 (15): 1516-25.

14. Lindberg JS, Culleton B, Wong G, et al. Cinacalcet $\mathrm{HCl}$, an oral calcimimetic agent for the treatment of secondary hyperparathyroidism in hemodialysis and peritoneal dialysis: a randomized, double-blind multicenter study. J Am Soc Nephrol 2005; 16: 800-07.

15. Moe S, Chertow GM, Coburn JW, et al. Achieving NFK$\mathrm{K} / \mathrm{DOQI}$ bone metabolism and disease treatment goals with Cinacalcet $\mathrm{HCl}$. Kidney Int 2005; 67: 760-71.

16. Goodman WG, Fadda GZ, Filkelstein FO, et al. Cinacalcet $\mathrm{HCl}$ is an effective primary therapy for the management of secondary hyperparathyroidism (HPT). J Am Soc Nephrol 2003; 14: 460A, SA-PO741.

17. Chertow GM, Blumenthal S, Turner S, et al. Cinacalcet hydrochloride (Sensipar) in hemodialysis patients on active vitamin D derivates with controlled PTH and elevated calcium and phosphorus. Clin J Am Soc Nephrol 2006; 1(2): 305-12.

18. Messa P, Macario F, Yaqoob M, et al. The OPTIMA study: assessing a new cinacalcet $\left(\right.$ Mimpara ${ }^{\circledR} /$ Sensipar $^{\circledR}$ ) treatment algorithm for secondary hyperparathyroidism. Clin J Am Soc Nephrol 2008; 3: 36-45.

19. Moe SM, Goodman WG, Cunningham J, et al. Cinacalcet $\mathrm{HCl}$ sustains long-term control of secondary hyperparathyroidism. Nephrol Dial Transplant 2005; 16: 501A, F-PO755.

20. Moe SM, Cunningham J, Brommer J, et al. Long-term treatment of secondary hyperparathyroidism with the calcimimetic cinacalcet $\mathrm{HCl}$. Nephrol Dial Transplant 2005; 20: 2168-93.

21. Chonchol M, Locatelli F, Abboud HE, et al. A randomized, double-blind, placebo-controlled study to assess the efficacy and safety of Cinacalcet $\mathrm{HCl}$ in participants with CKD not receiving dialysis. Am J Kidney Dis 2009; 53: 197-207.

22. Evenepoel P, Claes K, Kuypers DR, Debruyne F, Vanrenterghem Y. Parathyroidectomy after successful kidney transplantation: a single centre study. Nephrol Dial Transplant 2007; 22: 1730-7.

23. Evenepoel P, Claes K, Kuypers D, Maes B, Bammens B, Vanrenterghem Y. Natural history of parathyroid function and calcium metabolism after kidney transplantation: a single-centre study. Nephrol Dial Transplant 2004; 19:1281.

24. Kruse AE, Eisenberger U, Frey FJ, Mohaupt MG. The calcimimetic Cinacalcet normalizes serum calcium in renal transplant patients with persistent hyperparathyroidism. Nephrol Dial Transplant 2005; 20: 1311-4.

25. Serra AL, Schwarz AA, Wick FH, et al. Successful treatment of hypercalcemia with Cinacalcet in renal transplant recipients with persistent hyperparathyroidism. Nephrol Dial Transplant 2005; 20: 1315-9.

26. Szwarc I, Argilés A, Garrigue V, et al. Cinacalcet chloride is efficient and safe in renal transplant recipients with posttransplant hyperparathyroidism. Transplantation 2006; 82: 675-80.

27. Peacock M, Bilezikian JP, Klassen PS, et al. Cinacalcet hydrochloride maintains long-term normocalcemia in patients with primary hyperparathyroidism. J Clin Endocrinol Metab 2005; 90(1): 135-41.

28. Peacock M, Scumpia S, Bolognese MA, et al. Long-term control of primary hyperparathyroidism with cinacalcet. J Bone Miner Res 2006; 21 (Suppl 1): S38.

29. Silverberg SJ, Faiman C, Bilezikian JP, et al. The effects of Cinacalcet $\mathrm{HCl}$ (AMG 073) on serum calcium levels in patients with parathyroid carcinoma or recurrent primary hyperparathyroidism after parathyroidectomy. J Bone Miner Res 2003; 18: 171. 